\title{
Continuous Glucose Monitoring System in children with type 1 diabetes mellitus: a systematic review and meta-analysis
}

\author{
D. T. Golicki • D. Golicka • L. Groele • E. Pankowska
}

Received: 4 August 2007 / Accepted: 8 October 2007 / Published online: 1 December 2007

(C) Springer-Verlag 2007

\begin{abstract}
Aims/hypothesis We investigated the potential effects of the Continuous Glucose Monitoring System (CGMS), as compared with self-monitoring of blood glucose, on glycaemic control in children with type 1 diabetes.

Methods The following electronic databases were searched throughout June 2007: MEDLINE, EMBASE and The Cochrane Library. Additional references were obtained from reviewed articles. Only randomised controlled trials were included.

Results We included five trials involving 131 type 1 diabetic patients in the study. Combined data from all trials showed that the CGMS did not significantly reduce $\mathrm{HbA}_{1 \mathrm{c}}$ levels compared with control groups. The pooled weighted mean difference was $-0.02 \%(95 \% \mathrm{CI}-0.29$ to 0.25$)$ with a fixed model and remained insignificant in the random effect model. Sensitivity analysis determined that the findings were stable. There was a trend towards a longer time under the CGMS curve for glucose $<3.89 \mathrm{mmol} / 1$ in the CGMS group compared with the control group (mean difference $49.00 \mathrm{~min}, 95 \% \mathrm{CI}-18.00$ to 116.00 ). The CGMS significantly increased the number of insulin dose changes
\end{abstract}

Electronic supplementary material The online version of this article (doi:10.1007/s00125-007-0884-9) contains supplementary material, which is available to authorized users.

D. T. Golicki $(\bowtie)$

Department of Pharmacoeconomics,

Medical University of Warsaw,

ul. Pawinskiego 3A,

02-106 Warsaw, Poland

e-mail: dgolicki@amwaw.edu.pl

D. Golicka $\cdot$ L. Groele $\cdot$ E. Pankowska

Second Department of Paediatrics, Medical University of Warsaw,

Warsaw, Poland per patient per month for those managed with CGMS compared with the control groups (mean difference 6.3 changes, 95\% CI 2.88-9.72).

Conclusions/interpretation The Continuous Glucose Monitoring System is not better than self-monitoring of blood glucose with regard to improvement of metabolic control among type 1 diabetic children. However, due to the small number of participants and methodological limitations of the studies included, findings of this meta-analysis should be interpreted with caution.

Keywords Adolescent · CGMS · Child · Continuous glucose monitoring $\cdot$ Haemoglobin $\mathrm{A}_{1 \mathrm{c}} \cdot \mathrm{HbA}_{1 \mathrm{c}} \cdot$ Meta-analysis .

Randomised controlled trials · Type 1 diabetes mellitus

$\begin{array}{ll}\text { Abbreviations } \\ \text { CGM } & \text { continuous glucose monitoring } \\ \text { CGMS } & \text { Continuous Glucose Monitoring System } \\ \text { DCCT } & \text { Diabetes Control and Complications Trial } \\ \text { ITT } & \text { intention-to-treat } \\ \text { RCT } & \text { randomised controlled trial } \\ \text { RR } & \text { risk ratio } \\ \text { SMBG } & \text { self-monitoring of blood glucose } \\ \text { WMD } & \text { weighted mean difference }\end{array}$

\section{Introduction}

The Diabetes Control and Complications Trial (DCCT) indicated the importance of improving glucose control when seeking to reduce the risk of microvascular complications of type 1 diabetes [1-3]. For intensively treated participants in the DCCT, frequent glucose monitoring was considered an important factor for attaining better glucose control. 
Continuous glucose monitoring (CGM) provides maximal information about changes in blood glucose levels throughout the day and facilitates optimal treatment decisions for the diabetic patient [4]. Moreover, it provides information about the direction, magnitude, duration, frequency and the causes of fluctuations in blood glucose levels. Compared with conventional intensified glucose monitoring, defined as three to four blood glucose measurements per day, continuous monitoring provides much greater insight into glucose levels throughout the day [4].

There are several devices for CGM [5-10]. Generally, two types of CGM are distinguished. One is the Holter-type of glucose monitoring, where results are shown and analysed retrospectively; the second one uses real-time presentation of glycaemia values. The Continuous Glucose Monitoring System (CGMS; Medtronic Minimed, Northridge, CA, USA) was the first device to be approved and is the most utilised one, both in USA and Europe [5]. It provides a retrospective graphical view of the glycaemia profile measured during last three days.

Diabetes in children and adolescents is characterised by high glycaemia variability, tendency towards hypoglycaemia and difficulties in insulin adjustment. Moreover, children are often incapable of handling their self-monitoring and treatment responsibly. The management of this group of diabetic patients creates unique challenges. The need to protect them against long-term consequences of hypoglycaemia, as well as hyperglycaemia, clearly justifies the use of CGM. It is a useful tool in the diagnosis of asymptomatic nocturnal hypoglycaemia and in the clinical care of children with type 1 diabetes, but its effect on metabolic control remains controversial. Several studies, many of them strictly observational, have assessed the effect of CGM on metabolic control of type 1 diabetes [11-13]. We believe that a metaanalysis of all relevant studies available to date is important in order to establish the potential benefits of CGM. As effects of CGM seem to be device-specific, pooling data obtained by different systems might result in misleading conclusions. Hence, we decided to focus on just one continuous subcutaneous glucose monitoring device. Our choice of the CGMS was dictated by the fact that it was the first product on the market and used in most of the studies. In this work, we present results of the systematic review and meta-analysis.

\section{Methods}

Inclusion and exclusion criteria Systematic review and meta-analysis were conducted according to standards of the Cochrane Collaboration [14]. Studies included in the review had to be randomised controlled trials (RCTs) in which the CGMS and self-monitoring of blood glucose
(SMBG) were compared with SMBG alone in management of type 1 diabetes. Studies on other CGM devices were excluded. The studies had to be performed in children. The primary outcome measure was improvement in diabetes control according to $\mathrm{HbA}_{1 \mathrm{c}}$. The secondary outcome measures were: improvement in fructosamine, major hypoglycaemic episodes (as defined by the investigators), minor hypoglycaemic episodes, mean daily area under the CGMS curve for glucose $<3.89 \mathrm{mmol} / \mathrm{l}$, mean daily area over the CGMS curve for glucose $>9.99 \mathrm{mmol} / 1$, adjustments of insulin dose, local adverse effects and adherence. In addition to these outcomes, we also extracted other reported data, if relevant to our review.

Search strategy The following electronic databases were systematically searched for relevant studies: MEDLINE (1966-June 2007), EMBASE (1980-June 2007), The Cochrane Controlled Trials Register (issue 2, 2007) and The Cochrane Database of Systematic Reviews (issue 2, 2007). The text word terms and medical subject headings (MeSH) used were: 'diabetes type 1'; 'diabetes mellitus, type 1'; 'type 1 diabetes mellitus'; 'T1DM' and 'adolescent*'; 'child*'; 'infant*' and 'CGMS'; 'CGM*'; 'continuous glucose monitoring'. Subsequently, reference lists based on original studies and review articles were identified. Despite the lack of limitations with regard to the language of articles, specific types of publications (i.e. letters to the editor, abstracts and proceedings from scientific meetings) were eliminated from the study.

Data extraction Titles and abstracts identified according to the search strategy were screened independently by two reviewers (D. T. Golicki, D. Golicka). All potentially relevant articles were retained and their full text was examined to determine whether they meet the inclusion criteria. Data extraction was carried out independently by two reviewers (D. T. Golicki, D. Golicka) using standard data extraction forms. Extracted data were compared with eliminate errors. Discrepancies between the reviewers were resolved by discussion.

Study quality Reviewers independently, without blinding to authorship or journal, assessed the quality of the studies that met the inclusion criteria. Application of the following strategies associated with good quality studies was examined: (1) allocation concealment; (2) blinding of participants, outcome assessors and data analysts (yes/no/not reported); (3) intention-to-treat (ITT) analysis (yes/no); and (4) comprehensive follow-up. Allocation concealment was considered adequate when the randomisation method used did not allow the investigator or the participant to identify or influence the intervention group before the entry of eligible participants into the study. The quality of allocation 
concealment was regarded as unclear when randomisation was used, but no information about the method of randomisation was available. It was regarded as inadequate when inappropriate methods of randomisation (e.g. alternate medical record numbers, unsealed envelopes, tossing the coin) were used. In ITT analysis, a 'yes' answer meant that the authors had specifically reported undertaking this type of analysis and or/that our own study confirmed this finding. Conversely, 'no' meant that the authors did not report the use of ITT analysis and/or that we could not confirm its use in the study assessment. The completeness of patient follow-up was evaluated by determining the percentage of participants excluded or lost in follow-up. Only studies with $>80 \%$ follow-up were included. We defined the categories of risk of bias by the number of criteria judged inadequate in each study, i.e. low risk of bias (one or less inadequate criterion), medium risk of bias (three or less inadequate criteria) and high risk of bias (more than three inadequate criteria).

Statistical methods Two trials included in meta-analysis were crossover studies [15, 16]. According to Cochrane Group [14], 'paired' analysis is the most appropriate method of analysing crossover trials, where each patient acts as his or her own control. Yet, lack of individual patient data did not allow us to perform this kind of analysis. In effect, we decided to apply a different approach for incorporation of crossover trials, i.e. to include data only from the first period of trial. In the event of parallel groups trials, data from the end of the trial were used. Data were analysed using The Review Manager (Revman Computer program, Version 4.2.9 for Windows; The Nordic Cochrane Centre, Copenhagen, Denmark). The weighted mean difference (WMD) was selected to determine differences in continuous outcomes between the treatment and the control groups. Meta-analysis of continuous data using mean difference demanded extraction of the following: the mean values of the outcomes, the standard deviations of the outcomes and the number of participants in both groups for whom the outcome was assessed. In one study [17], data concerning the effect of treatment on $\mathrm{HbA}_{1 \mathrm{c}}$ were calculated on the basis of individual patient results, presented in the article in form of a table. Yet, these values differed from the summarised results presented by the authors. In another study [18], standard deviations of $\mathrm{HbA}_{1 \mathrm{c}}$ changes were obtained from $95 \%$ CIs, using the formula: $\mathrm{SD}=\sqrt{ } N \times$ (upper limit-lower limit)/3.92 [14]. Two trials [15, 16] did not report data on standard deviations of changes in $\mathrm{HbA}_{1 \mathrm{c}}$. Missing information was sought from the authors without success. As exclusion of both trials from the analysis might have led to bias, we decided to impute standard deviations for the changes from baseline, based on all available data from other trials, using a method described in the Cochrane reviewers' handbook [14]. The binary measure for individual studies and pooled statistics was calculated as the risk ratio (RR) between the experimental and control groups with $95 \%$ CI. The difference between study groups was considered significant when the $p$ value was $<0.05$ or when the $95 \%$ CI for RR did not exceed 1.0 and that for WMD did not exceed 0 (equivalent to $p<0.05$ ). The weight given to each study was based on variance inversion. Heterogeneity was determined by $\chi^{2}$ and $I^{2}$, which stand for the percentage of total variations between studies that are attributed to heterogeneity rather than chance. Sensitivity analysis, on the data assessed directly after the use of CGMS, was performed excluding the two trials [15, 16] with imputed standard deviations.

\section{Results}

Description of studies Initially, we identified 25 articles that underwent further examination. Table 1 summarises characteristics of the included trials. Five RCTs involving 131 participants met our predefined inclusion criteria [1519]; the remaining 20 studies were excluded [7, 11-13, 2035]. Table 2 summarises the characteristics of excluded trials, including reasons for exclusion.

All included trials were small, one-centre studies; two of them were performed in Europe $[15,16]$, two in the USA $[17,19]$ and one in Australia [18]. Three of the included trials [17-19] had parallel-group design and two others [15, 16] were crossover. There was considerable clinical heterogeneity among the trials with regard to the children's age, diabetes duration, frequency of CGMS use and insulin adjustments. Depending on the trial, patients had to wear CGMS: every 5 days [17], every 2 weeks [16], every 3 weeks [18], every 2 months [19] or once during a 3 month period [15]. We also found significant differences in the frequency of insulin changes: $>11$ times during 1 month (experimental group only) [17], every 3 weeks [18], every 6 weeks [16] and every 2 months [19]. Follow-up periods extended up to 3 months in three trials [15-17] and up to 6 months in the other two trials $[18,19]$.

The methodological quality of the studies varied [see Electronic supplementary material (ESM) Table 1]. We assessed a risk of low bias in only one trial [19]. Only two trials [18, 19] contained information on the method of generating allocation sequences. In both cases, the method was adequate. Two trials $[15,16]$ were described as doubleblinded, one [19] as single-blinded and two [17, 18] were open. In one trial [16], the authors reported undertaking an ITT analysis, but our own survey did not confirm this finding. Withdrawals and dropouts were described adequately in all studies. All trials contained a sufficient proportion $(\geq 80 \%)$ of participants in the final analysis; two 


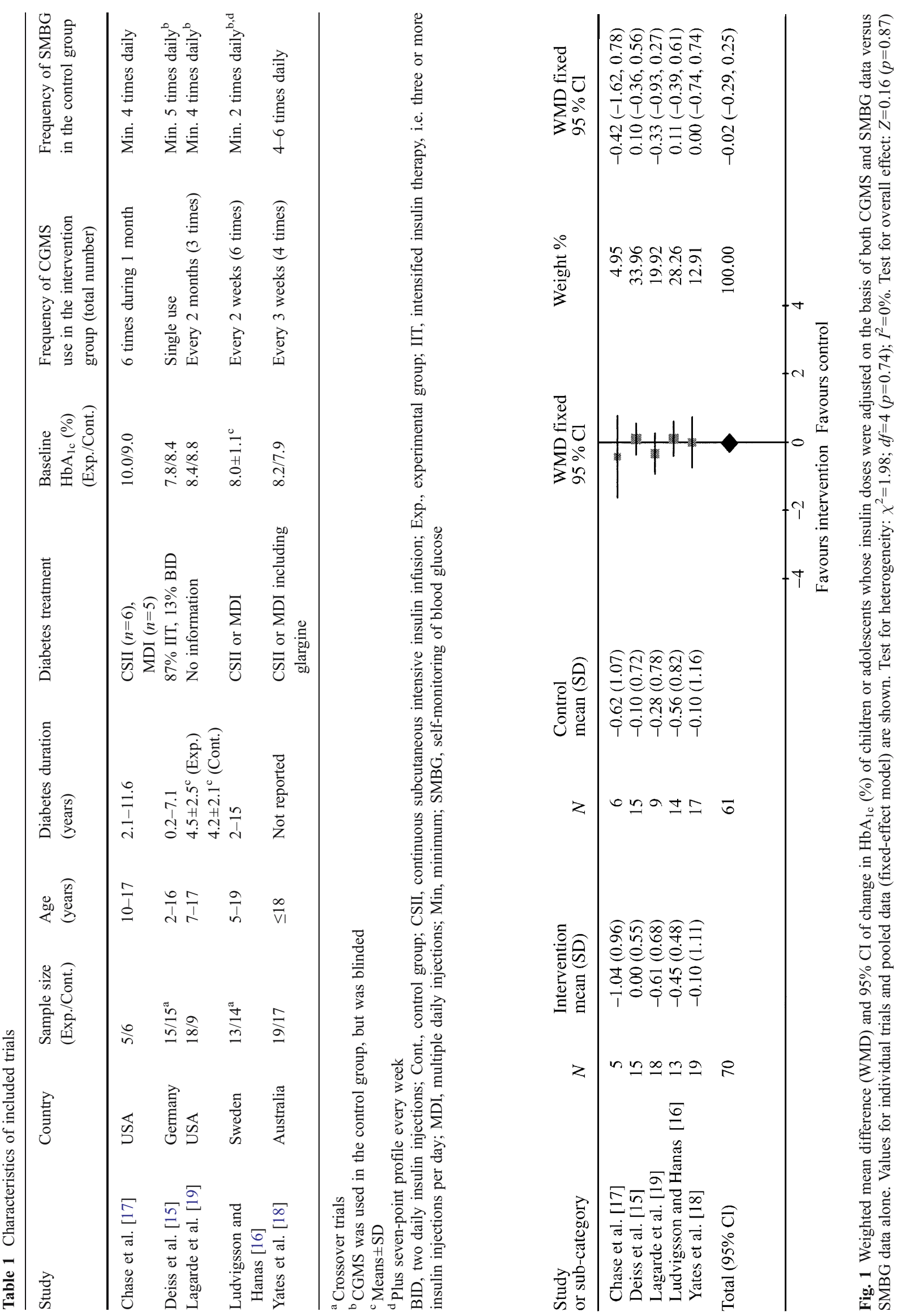


Table 2 Characteristics of excluded studies

\begin{tabular}{ll}
\hline Study & Reason(s) for exclusion \\
\hline Bode et al. [20] & Non-randomised, uncontrolled trial \\
Bode et al. [7] & RCT performed in adults. Guardian Continuous Monitoring System used \\
Boland et al. [21] & Non-randomised, uncontrolled trial \\
Burdick et al. [22] & Non-randomised, uncontrolled trial. Real-Time glucose sensing system used \\
Chase et al. [23] & RCT; GlucoWatch G2 Biographer used \\
Chico et al. [24] & Non-randomised, uncontrolled trial \\
Deiss et al. [25] & Non-randomised, uncontrolled trial \\
Deiss et al. [26] & RCT; Guardian RT system used \\
DirectNet [27] & RCT; GlucoWatch G2 Biographer used \\
DirectNet [28] & RCT; GlucoWatch G2 Biographer used \\
Fiallo-Scharer et al. [29] & RCT; GlucoWatch G2 Biographer used \\
Garg et al. [30] & RCT; DexCom STS system used \\
Halvorson et al. [31] & Non-randomised, uncontrolled trial. Paradigm Real-Time system used \\
Kaufman et al. [11] & Non-randomised, uncontrolled trial \\
Salardi et al. [12] & Non-randomised, uncontrolled trial \\
Schaepelynck-Belicar et al. [13] & Non-randomised, uncontrolled trial \\
Tanenberg et al. [32] & Randomised controlled trial performed in adults \\
Tansey et al. [33] & Non-randomised study; did not report any of our defined outcomes \\
Weinstein et al. [34] & Non-randomised, uncontrolled trial. FreeStyle Navigator system used \\
Wilson et al. [35] & Non-randomised, uncontrolled trial. FreeStyle Navigator system used \\
\hline &
\end{tabular}

$[15,19]$ of them included all $(100 \%)$ of the initially selected participants.

$H b A_{1 c}$ Five papers contained data on $\mathrm{HbA}_{1 \mathrm{c}}$ improvement [15-19]. Meta-analysis of five RCTs (131 participants) showed no reduction in $\mathrm{HbA}_{1 \mathrm{c}}$ (WMD $-0.02 \%, 95 \% \mathrm{CI}-0.29$ to 0.25 ; $p=0.87$ ) for patients managed with CGMS and SMBG compared with patients monitored with SMBG alone (Fig. 1; ESM Table 2). Adjustment of the meta-analysis model from fixed to random effects did not influence the outcome results. The included studies were homogenous $\left(\chi^{2}=1.98\right.$, $\left.p=0.74, I^{2}=0 \%\right)$. The main findings were stable in sensitivity analysis based on data obtained directly after CGMS use (WMD $-0.05 \%$, 95\% CI -0.27 to $0.17 ; p=$ $0.65)$ and after excluding the two trials $[15,16]$ with imputed standard deviations (WMD $-0.23 \%, 95 \% \mathrm{CI}-0.66$ to $0.21 ; p=0.30)$.

Fructosamine The reported outcome was based on results from only one RCT [18]. Findings showed no reduction of fructosamine between the CGMS and the control group at 6 weeks (mean difference $0.5 \mu \mathrm{mol} / 1,95 \% \mathrm{CI}-40.96$ to $41.96 ; p=0.98$ ) and at 12 weeks (mean difference $-7.2 \mu \mathrm{mol} / 1$, $95 \% \mathrm{CI}-55.57$ to $41.17 ; p=0.77)$.

Major hypoglycaemic episodes No severe hypoglycaemic events occurred in either the CGMS or the control groups in any study included in the review.

Minor hypoglycaemic episodes For this outcome, based on the results from only one trial [19], no difference was seen in the number of minor hypoglycaemic episodes during the total CGMS-monitored period between the CGMS and the control group (mean difference $0.53,95 \% \mathrm{CI}-0.68$ to 1.74 ; $p=0.39$ ).

Mean daily time and daily area under the CGMS curve for glucose $<3.89 \mathrm{mmol} / \mathrm{l}$ The only RCT [19] $(n=27)$ to report this outcome demonstrated a trend towards a longer time under the CGMS curve for glucose $<3.89 \mathrm{mmol} / \mathrm{l}$ in the CGMS group than in the control group (mean difference $49.00 \mathrm{~min}, 95 \% \mathrm{CI},-18.00$ to $116.00 ; p=0.15)$. The same study reported no significant difference in the daily area under the CGMS curve for glucose $<3.89 \mathrm{mmol} / 1$ between the CGMS and the control group (mean difference $35.85 \mathrm{mmol} \mathrm{l}^{-1} \mathrm{~min}^{-1}$ per day, $95 \% \mathrm{CI}-28.58$ to 100.29 ; $p=0.28)$.

Daily area over the CGMS curve for glucose $>9.99 \mathrm{mmol} / \mathrm{l}$ The only RCT [19] to report on this outcome noted no difference in the daily area over the CGMS curve for glucose $>9.99 \mathrm{mmol} / \mathrm{l}$ between the CGMS and the control group (mean difference $0.33 \mathrm{mmol} 1^{-1} \mathrm{~min}^{-1}$ per day, 95\% CI -10.26 to $10.92 ; p=0.95)$.

Ketoacidosis A single participant in one trial [18] was admitted to hospital with ketoacidosis. The patient was from the CGMS group. Ketoacidosis was thought to be due to insulin omission.

Adjustments of insulin dose Two papers included information on insulin dose adjustments. One trial [17] showed an 
increase in the number of insulin dose changes per patient per month for those managed with CGMS compared with the control group (mean difference 6.3 changes, 95\% CI 2.88 to $9.72 ; p=0.0003)$. The second trial [15] demonstrated an increase by $35 \%$ in the relative chance of insulin dose changes in the CGMS open group compared with the CGMS-blinded group (95\% CI $0.98-1.85 ; p=0.07)$.

Local adverse events and adherence A narrative synthesis of the data on local adverse events and adherence was undertaken. Among the five trials included in the review, local adverse effects were reported only in two $[15,18]$. Deiss et al. [15] observed mild local side-effects like redness at the application site in $21(23 \%)$ cases, redness and itching in 14 cases (16\%), and painful redness in one case. None of these complaints led to a preterm removal of CGMS. Yates et al. [18] reported that one participant on continuous subcutaneous intensive insulin infusion withdrew after $12 \mathrm{~h}$ of CGMS due to skin irritation at the sensor site.

\section{Discussion}

The main findings of our meta-analysis show that the CGMS is just as suitable as self-monitoring of blood glucose levels when it comes to improving $\mathrm{HbA}_{1 \mathrm{c}}$ in children with type 1 diabetes. Findings were stable with both fixed and random effect models, and in sensitivity analysis. We found a trend towards a longer time under the CGMS curve for glucose $<3.89 \mathrm{mmol} / \mathrm{l}$ in the CGMS group than in the control group. CGMS also significantly increased the number of insulin dose changes per patient per month.

Every meta-analysis is only as good as the constituent studies. All but one of the trials included in our analysis had methodological limitations, including unclear or inadequate allocation concealments, lack of ITT analysis and no blinding. In the only trial with an estimated low risk of bias [19], participants in the intervention group were significantly younger than those in the control group. In all trials, small study sample sizes were also limiting. The overall quality of studies was low, especially compared with typical trials in drug development.

Meta-analysis trials included two crossover studies [15, 16]. The Cochrane Group reported [14] that the most suitable method for analysing crossover trials is 'paired' analysis, where each patient acts as his/her own control. Due to the lack of individual patient data, we were unable to perform this kind of analysis. As a result, the second most common approach was applied, with inclusion of data from the first trial period. Another difficulty with the crossover trials $[15,16]$ was due to the lack of data on standard deviations of $\mathrm{HbA}_{1 \mathrm{c}}$ changes. Moreover, requests made directly to the authors failed to provide the missing information. As elimination of both trials from the analysis might have led to bias, we decided to assign standard deviations to changes from baseline from all other trials with available data, using the method proposed in the Cochrane Handbook [14]. Estimated standard deviations were small, confidence intervals narrow and trials were over-weighted in pooled analysis. Despite this, the main findings were stable in sensitivity analysis, excluding those trials with imputed standard deviations.

No formal attempt to search for publication bias was made, as only a few studies on any given endpoint were reported and any formal method would have had little power. To reduce the risk of publication bias, language restrictions were abolished. An extensive search strategy for the identification of published trials was made; yet, we did not attempt to identify unpublished trials.

Although studies included in the meta-analysis were not statistically heterogeneous, they varied in the clinical context of CGMS use and timing of insulin adjustments. Depending on the trial, patients had to carry out CGMS: every 5 days [17], every 2 weeks [16], every 3 weeks [18], every 2 months [19] or even once in 3 months [15]. Variations in the frequency of insulin changes were noted: over 11 times during 1 month [17], every 3 weeks [18], every 6 weeks [16] and every 2 months [19]. There seems to be no standard way of using CGMS in order to improve the metabolic control of diabetes.

The analysis conducted by us was limited to only one device, namely the Continuous Glucose Monitoring System (Medtronic MiniMed). There are several CGM devices, which differ in terms of sensor type, mechanism and location, frequency of testing and blood glucose data presentation (retrospective or real time). The fact that access to the CGMS data is retrospective can be regarded as a major technical limitation and explains, at least in part, the negative outcome of the trials included. It cannot be ruled out that other devices, especially real-time systems with alarm options or integrated insulin pumps, could improve the metabolic control of type 1 diabetes. In time, the Holter-type CGM will probably be replaced by the real-time CGM in diabetic practice, but can still remain a useful tool for conducting research, in which new parameters, such as mean amplitude of glycaemic excursions, variability of glucose and duration of hypoglycaemia can be considered as risk factors for late complications.

The limitations discussed suggest steps towards improving the quality of research in this area. Further well-conducted clinical studies should have more participants, probably parallel group rather than crossover design and longer than 6 month observation periods. Future studies should also address the question of establishing a standard protocol on the basis of optimum efficiency for the use of CGMS. 
Conclusion The CGMS did not prove more advantageous than self-monitoring of blood glucose levels in improving metabolic control of type 1 diabetic children. Nonetheless, results of meta-analysis should be regarded with caution, due to the small study group sizes and methodological limitations of the included studies.

Acknowledgements The authors thank H. Szajewska for her critical review of the manuscript and valuable remarks.

Duality of interest E. Pankowska has received speaker's honoraria from Medtronic MiniMed. This study was fully funded by the Medical University of Warsaw, Warsaw, Poland. Otherwise the authors declare that there is no duality of interest associated with this manuscript.

\section{References}

1. The Diabetes Control and Complications Trial Research Group (DCCT) (1993) The effect of intensive treatment of diabetes on the development and progression of long term complications in insulin-dependent diabetes mellitus. N Engl J Med 329:977-986

2. The DCCT Research Group (1994) Effect of intensive diabetes treatment on the development and progression of long-term complications in adolescents with insulin-dependent diabetes mellitus: Diabetes Control and Complications Trial. J Pediatr 125:177-188

3. The Diabetes Control and Complications Trial/Epidemiology of Diabetes Interventions and Complications Research Group (2000) Retinopathy and nephropathy in patients with type 1 diabetes four years after a trial of intensive therapy. N Engl J Med 342:381-389

4. Klonoff DC (2005) Continuous glucose monitoring: roadmap for 21st century diabetes therapy. Diabetes Care 28:1231-1239

5. Gross TM, Bode BW, Einhorn D et al (2000) Performance evaluation of the MiniMed continuous glucose monitoring system during patient home use. Diabetes Technol Ther 2:49-56

6. Potts RO, Tamada JA, Tierney MJ (2002) Glucose monitoring by reverse iontophoresis. Diabetes Metab Res Rev 18(Suppl 1): S49-S53

7. Bode B, Gross K, Rikalo N et al (2004) Alarms based on real-time sensor glucose values alert patients to hypo- and hyperglycemia: the guardian continuous monitoring system. Diabetes Technol Ther 6:105-113

8. Maran A, Crepaldi C, Tiengo A et al (2002) Continuous subcutaneous glucose monitoring in diabetic patients: a multicenter analysis. Diabetes Care 25:347-352

9. Pfuetzner A, Caduff A, Larbig M, Schrepfer T, Forst T (2004) Impact of posture and fixation technique on impedance spectroscopy used for continuous and noninvasive glucose monitoring. Diabetes Technol Ther 6:435-441

10. Feldman B, Brazg R, Schwartz S, Weinstein R (2003) A continuous glucose sensor based on wired enzyme technology: results from a 3-day trial in patients with type 1 diabetes. Diabetes Technol Ther 5:769-779

11. Kaufman FR, Gibson LC, Halvorson M, Carpenter S, Fisher LK, Pitukcheewanont P (2001) A pilot study of the continuous glucose monitoring system: clinical decisions and glycemic control after its use in pediatric type 1 diabetic subjects. Diabetes Care 24:2030-2034

12. Salardi S, Zucchini S, Santoni R et al (2002) The glucose area under the profiles obtained with continuous glucose monitoring system relationships with $\mathrm{HbA}(\mathrm{lc})$ in pediatric type 1 diabetic patients. Diabetes Care 25:1840-1844

13. Schaepelynck-Belicar P, Vague P, Simonin G, Lassmann-Vague V (2003) Improved metabolic control in diabetic adolescents using the continuous glucose monitoring system (CGMS). Diabetes Metab 29:608-612

14. Higgins JPT, Green S (eds) (2007) Cochrane handbook for systematic reviews of interventions 4.2.6 (updated September 2006). The Cochrane Library, Issue 2. Wiley, Chichester

15. Deiss D, Hartmann R, Schmidt J, Kordonouri O (2006) Results of a randomised controlled cross-over trial on the effect of continuous subcutaneous glucose monitoring (CGMS) on glycaemic control in children and adolescents with type 1 diabetes. Exp Clin Endocrinol Diabetes 114:63-67

16. Ludvigsson J, Hanas R (2003) Continuous subcutaneous glucose monitoring improved metabolic control in pediatric patients with type 1 diabetes: a controlled crossover study. Pediatrics 111:933-938

17. Chase HP, Kim LM, Owen SL et al (2001) Continuous subcutaneous glucose monitoring in children with type 1 diabetes. Pediatrics 107:222-226

18. Yates K, Hasnat Milton A, Dear K, Ambler G (2006) Continuous glucose monitoring-guided insulin adjustment in children and adolescents on near-physiological insulin regimens: a randomized controlled trial. Diabetes Care 29:1512-1517

19. Lagarde WH, Barrows FP, Davenport ML, Kang M, Guess HA, Calikoglu AS (2006) Continuous subcutaneous glucose monitoring in children with type 1 diabetes mellitus: a single-blind, randomized, controlled trial. Pediatr Diabetes 7:159-164

20. Bode BW, Gross TM, Thornton KR, Mastrototaro JJ (1999) Continuous glucose monitoring used to adjust diabetes therapy improves glycosylated hemoglobin: a pilot study. Diabetes Res Clin Pract 46:183-190

21. Boland E, Monsod T, Delucia M, Brandt CA, Fernando S, Tamborlane WV (2001) Limitations of conventional methods of self-monitoring of blood glucose: lessons learned from 3 days of continuous glucose sensing in pediatric patients with type 1 diabetes. Diabetes Care 24:1858-1862

22. Burdick J, Chase P, Faupel M, Schultz B, Gebhart S (2005) Realtime glucose sensing using transdermal fluid under continuous vacuum pressure in children with type 1 diabetes. Diabetes Technol Ther 7:448-455

23. Chase HP, Beck R, Tamborlane W et al (2005) A randomized multicenter trial comparing the GlucoWatch biographer with standard glucose monitoring in children with type 1 diabetes. Diabetes Care 28:1101-1106

24. Chico A, Vidal-Rios P, Subira M, Novials A (2003) The continuous glucose monitoring system is useful for detecting unrecognized hypoglycemias in patients with type 1 and type 2 diabetes but is not better than frequent capillary glucose measurements for improving metabolic control. Diabetes Care 26:1153-1157

25. Deiss D, Hartmann R, Hoeffe J, Kordonouri O (2004) Assessment of glycemic control by continuous glucose monitoring system in 50 children with type 1 diabetes starting on insulin pump therapy. Pediatr Diabetes 5:117-121

26. Deiss D, Bolinder J, Riveline JP et al (2006) Improved glycemic control in poorly controlled patients with type 1 diabetes using realtime continuous glucose monitoring. Diabetes Care 29:2730-2732

27. Diabetes Research in Children Network (DirecNet) Study Group (2006) Psychological aspects of continuous glucose monitoring in pediatric type 1 diabetes. Pediatr Diabetes 7:32-38

28. Diabetes Research in Children Network (DirecNet) Study Group (2005) Youth and parent satisfaction with clinical use of the GlucoWatch G2 Biographer in the management of pediatric type 1 diabetes. Diabetes Care 28:1929-1935

29. Fiallo-Scharer R; Diabetes Research in Children Network Study Group (2005) Eight-point glucose testing versus the continuous 
glucose monitoring system in evaluation of glycemic control in type 1 diabetes. J Clin Endocrinol Metab 90:3387-3391

30. Garg S, Zisser H, Schwartz S et al (2006) Improvement in glycemic excursions with a transcutaneous, real-time continuous glucose sensor: a randomized controlled trial. Diabetes Care 29:44-50

31. Halvorson M, Carpenter S, Kaiserman K, Kaufman FR (2007) A pilot trial in pediatrics with the sensor-augmented pump: combining real-time continuous glucose monitoring with the insulin pump. J Pediatr 150:103-105

32. Tanenberg R, Bode B, Lane W et al (2004) Use of the Continuous Glucose Monitoring System to guide therapy in patients with insulin-treated diabetes: a randomized controlled trial. Mayo Clin Proc 79:1521-1526
33. Tansey MJ, Beck RW, Buckingham BA et al; The Diabetes Research in Children Network (DirecNet) Study Group (2005) Accuracy of the modified Continuous Glucose Monitoring System (CGMS) sensor in an outpatient setting: results from a diabetes research in children network (DirecNet) study. Diabetes Technol Ther 7:109-114

34. Weinstein RL, Schwartz SL, Brazg RL, Bugler JR, Peyser TA, McGarraugh GV (2007) Accuracy of the 5-day FreeStyle Navigator Continuous Glucose Monitoring System: comparison with frequent laboratory reference measurements. Diabetes Care 30:1125-1130

35. Wilson DM, Beck RW, Tamborlane WV et al; DirecNet Study Group (2007) The accuracy of the FreeStyle Navigator continuous glucose monitoring system in children with type 1 diabetes. Diabetes Care 30:59-64 